\title{
ON PROPERTIES POSSESSED BY SOLVABLE AND NILPOTENT GROUPS
}

\author{
CHRISTINE AYOUB 1
}

(Received 5 June 1967)

The object of this note is to study two properties of groups, which we will denote by $\left({ }^{*}\right)$ and $\left({ }^{* *}\right)$. The property $\left({ }^{*}\right)$ is possessed by solvable groups (and in fact, by groups which have a solvable invariant system) and the property $(* *)$ is possessed by nilpotent groups (and in fact, by groups which have a central system).

It is quite easy to show that if a group satisfies $\left(^{*}\right)$ locally, then it satisfies $\left(^{*}\right)$; this gives a short proof of Malcev's theorem that a locally solvable group cannot be simple unless it is cyclic of prime order. It should be remarked, however, that the proof given is simply an adaption of Malcev's proof - its only virtue is that it is short and easy.

Theorem 2 states that a finitely generated group $G$ satisfying $\left(^{*}\right)$ and the minimum condition for normal subgroups is finite and solvable, and Theorem 3 studies the connection between property $\left({ }^{*}\right)$ and a property studied by Ore.

Theorem 5 states that if the group $G$ - with hypercentre $C$ - satisfies (**), then $G / C$ satisfies (**); from this we deduce that if $G$ satisfies $\left({ }^{* *}\right)$ and the minimum condition for normal subgroups, $G$ is hypercentral.

\section{Notations}

$[a, b] \quad=a^{-1} b^{-1} a b$.

$n(U) \quad=$ normalizer of the subgroup $U$ in $G$.

$Z(G) \quad=$ centre of the group $G$.

$A \leqq B:=: A$ is a subgroup of $B$.

$A<B:=: A$ is a proper subgroup of $B$.

$A \triangleleft B:=: A$ is a normal subgroup of $B$.

$E \quad=$ trivial subgroup (consisting of the identity element).

Following Kurosh we call $G$ an $S I$-group ( $S N$-group) if it has an invariant (normal) system with abelian factors (see Kurosh [5, p. 171-73

1 This paper was written while the author held an NSF Science Faculty Fellowship. 
and p. 182]), and we call $G$ a $Z$-group if it has a central system - see Kurosh [5, p. 218]. We say that $G$ is a $Z A$-group if the upper central chain for $G$, possibly continued transfinitely, leads up to $G$ - see Kurosh [5, p. 218-19]. (Baer calls such a group hypercentral and uses the equivalent definition that $G$ is hypercentral if every epimorphic image $(\neq E)$ has a non-trivial centre.) $G$ is an $S I^{*}$-group if it has a solvable ascending invariant series (this is what Baer calls hyperabelian; again an equivalent definition is that the group $G$ is hyperabelian if every epimorphic image $(\neq E)$ has a nontrivial normal abelian subgroup).

\section{The property (*)}

Definition 1 . The group $G$ satisfies $\left(^{*}\right)$ if : given elements $a, b(\neq 1,1)$ in $G$, there is a normal subgroup $C=C(a, b)$ of $G$ such that $[a, b]$ is in $C$ but not both $a$ and $b$ are in $C$. define

REMARK. If $G$ satisfies $\left({ }^{*}\right)$, and $a, b(\neq 1,1)$ are elements of $G$, we can

$$
C_{a, b}=\{\cap C \mid C \triangleleft G,[a, b] \in C \text { and not both } a \text { and } b \text { are in } C\} .
$$

Clearly $C_{a, b}$ is normal in $G,[a, b]$ is in $C_{a, b}$ but not both $a$ and $b$ are in $C_{a, b}$. $C_{a, b}$ is the unique smallest normal subgroup of $G$ with these properties.

LEMma 1. (i) If $S$ is a subgroup of the group $G$ and if $G$ satisfies (*), then $S$ satisfies $\left({ }^{*}\right)$.

(ii) If $N$ is a normal subgroup of the group $G$ and if $G$ satisfies (*), then given elements $a, b(\neq 1,1)$ in $N$ there exists a normal subgroup $C$ of $G$ such that $C<N,[a, b] \in C$ and not both $a$ and $b$ are in $C$.

Thus if $G$ has a local system each of whose subgroups satisfies $\left({ }^{*}\right)$, the finitely generated subgroups of $G$ satisfy $\left(^{*}\right)$.

Proposition 1. If $G$ is an SI-group, then $G$ satisfies (*). In particular, if $G$ is solvable, $G$ satisfies $\left({ }^{*}\right)$.

Proof. Let $\Sigma$ be an invariant system for $G$ with abelian factors. Let $a, b(\neq 1,1)$ be any two elements of $G$ and define

$$
\begin{aligned}
& \bar{C}=\{\cap N \mid N \in \Sigma, a \text { and } b \text { both } \in N\}, \text { and } \\
& C=\{\cup K \mid K \in \Sigma, \text { not both } a \text { and } b \in K\} .
\end{aligned}
$$

Then $C<\bar{C}$ is a jump in $\Sigma$; hence $C / C$ is abelian so that $[a, b] \in C$. Clearly $C$ is a normal subgroup of $G$ and not both $a$ and $b$ belong to $C$.

Proposition 2. Let $G$ be a group and assume that for each pair of elements $a, b(\neq 1,1)$ a normal subgroup $C_{a, b}$ can be chosen so that $[a, b] \in C_{a, b}$, but not both $a$ and $b$ are in $C_{a, b}$ and that in addition these subgroups can be chosen 
in such a way that for $a, b(\neq 1,1), c, d(\neq 1,1)$ in $G$ either $C_{a, b} \leqq C_{c, d}$ or $C_{c, a} \leqq C_{a, b}$ (i.e. in such a way that the subgroups are linearly ordered). Then $G$ is an $S I$-group.

Proof. Complete the system of normal subgroups $\left\{C_{a, b}\right\}$ to a system $\Sigma$. We show that if $K<L$ is a jump in $\Sigma$, then $L / K$ is abelian. For suppose not; then there are elements $a$ and $b$ in $L$ with $[a, b]$ not in $K$. Now if $L \leqq C_{a, b}$, $a$ and $b$ both lie in $C_{a, b}$, which is impossible. Hence $C_{a, b}<L$, which implies that $C_{a, b} \leqq K$. But then $[a, b] \in K$, a contradiction.

THEOREM 1. If the group $G$ satisfies $\left({ }^{*}\right)$ locally, then $G$ satisfies $(*)$.

Proof. Let $\Sigma$ consist of all finitely generated subgroups of $G$. For $A$ in $\Sigma$ and $a, b(\neq 1,1)$ in $A$ let $C_{a, b}(A)$ be a fixed normal subgroup of $A$ such that $a$ and $b$ are not both in $C_{a, b}(A)$ but $[a, b] \in C_{a, b}(A)$.

For $a, b(\neq 1,1)$ in $G$ and $S$ a finite subset of $G$ define

$$
K_{a, b}(S)=\left\{\cap C_{a, b}(A) \mid A \in \Sigma,\{a, b, S\} \subseteq A\right\} .
$$

Clearly if $S_{1} \subseteq S_{2}$ are finite subsets of $G, K_{a, b}\left(S_{1}\right) \leqq K_{a, b}\left(S_{2}\right)$. Thus for arbitrary finite subsets $S_{1}$ and $S_{2}$ of $G, K_{a, b}\left(S_{i}\right) \leqq K_{a, b}\left(S_{1} \cup S_{2}\right)$ for $i=1,2$.

Let $H_{a, b}=\left\{\cup K_{a, b}(S) \mid S\right.$ a finite subset of $\left.G\right\}$. It is clear that $H_{a, b}$ is a subgroup of $G$ which contains $[a, b]$ but does not contain both $a$ and $b$. It remains to verify that $H_{a, b}$ is normal in $G$. So let $c \in H_{a, b}$ and $d \in G$. Then $c \in K_{a, b}(S)$ for some finite subset $S$ of $G$ and we can assume that $d \in S$. Now $c \in C_{a, b}(A)$ for each $A$ in $\Sigma$ with $\{a, b, S\} \subseteq A$. Hence by the normality of $C_{a, b}(A)$ in $A, d^{-1} c d$ is in $C_{a, b}(A)$ for each $A$ in $\Sigma$ with $\{a, b, S\} \subseteq A$. Hence $d^{-1} c d \in K_{a, b}(S)$ and this implies that $d^{-1} c d \in H_{a, b}$.

COROLLARY I. If $G$ is locally solvable and not cyclic of prime order, then $G$ is not simple.

As noted in the introduction the proof of Theorem 1 is just Malcev's proof adapted to the case considered. Malcev's Theorem states that if a group has the property $S I$ locally then it is an $S I$-group. For a proof see Kurosh [5, p. 183-87].

DEFINITION 2. Let $V$ be a maximal normal subgroup of the group $U$; then $U / V$ is a tor of $U$.

LEMMA 2. Let $G$ be a group which satisfies $\left(^{*}\right)$ and the minimum condition for normal subgroups. Then if $K$ is a normal subgroup of $G$, any tor of $K$ is abelian.

Proof. Assume that the lemma is false and let $U$ be a minimal normal subgroup of $G$ with a non-abelian tor. ${ }^{2}$ Hence there exists $V \triangleleft U$ such that

2 i.e. $U$ is a normal subgroup of $G$, has a non-abelian tor and is minimal with respect to this property. 
$U / V$ is simple non-abelian. Thus there exist elements $a$ and $b$ in $U$ such that $[a, b] \notin V$. Let $C$ be a normal subgroup of $G$ such that $C<U,[a, b] \in C$ and not both $a$ and $b \in C$. Then $V \leqq V C \leqq U$ and $V \neq V C$ since $[a, b] \in C$ but $[a, b] \notin V$. Hence by the maximality of $V, U=V C$.

Now $U / V=V C / V \cong C / V \cap C$. Thus $C$ is a normal subgroup of $G$ with a non-abelian tor and $C<U$. This contradicts the minimality of $U$.

THEOREM 2. Let $G$ be a finitely generated group which satisfies $\left(^{*}\right)$ and the minimum condition for normal subgroups. Then $G$ is a finite, solvable group.

Proof. Let $K$ be a normal subgroup of $G$ and assume $K$ is minimal such that $G / K$ is finite and solvable. Assume $K \neq E$. Then since $K$ is finitely generated, it possesses a maximal normal subgroup $M$. By Lemma $2, K / M$ is abelian and hence cyclic of prime order. Let $\bar{M}=\left\{\cap M^{x} \mid x \in G\right\}$. Since $M$ is of finite index in $G, \bar{M}$ is also of finite index in $G$. Furthermore, $\bar{M}$ is normal in $G$ and $G / \bar{M}$ is solvable since $K / \bar{M}$ is solvable. But $\bar{M}<K$ so that the minimality of $K$ is contradicted. Hence $K=E$ and $G$ is finite and solvable.

Corollary 2. Let $G$ be a group which satisfies $\left({ }^{*}\right)$ and the minimum condition for subgroups $U$ such that $n(U)>U$. Then $G$ is locally finite and locally solvable. Furthermore, $G$ is an $S I^{*}$-group.

Proof. If $H$ is a finitely generated subgroup of $G, H$ satisfies (*) and the minimum condition for normal subgroups. Hence $H$ is finite and solvable. In particular, $H$ is an $S I$-group. By the local theorem for $S I$-groups, $G$ is an $S I$-group and by the minimum condition for normal subgroups, $G$ is an $S I^{*}$-group.

We now consider a property which Kurosh denotes by $(Q)$, and a somewhat weaker one which will be denoted by $\left(Q^{\prime}\right)$. The property $(Q)$ was introduced by Ore (see Kurosh [5, p. 181] and Ore [7, p. 251, Theorem 9]).

Definition 3. The subgroup $A$ of $G$ is almost normal in $G$ if there exists a normal subgroup $N$ of $G$ such that $G=A N$ and $A \cap N \triangleleft G$.

Definition 4. The group $G$ satisfies ( $Q$ ) if $A<B \leqq G$, and $A$ maximal in $B$, implies that $A$ is almost normal in $B$.

Definition 5. The group $G$ satisfies $\left(Q^{\prime}\right)$ if $A<B \leqq G$, and $A$ maximal in $B$, implies that either $A \triangleleft B$, or there exists a proper normal subgroup $N$ of $B$ such that $B=A N$.

It is clear that if $G$ satisfies $(Q)$, it satisfies $\left(Q^{\prime}\right)$.

THEOREM 3. (i) If the group $G$ satisfies $(*)$, it satisfies $\left(Q^{\prime}\right)$.

(ii) If the group $G$ satisfies $\left(^{*}\right)$ and the minimum condition for subgroups $U$ such that $n(U)>U$, then $G$ satisfies $(Q)$. 
(iii) If the group $G$ satisfies $\left(Q^{\prime}\right)$ and the minimum condition for subgroups, then $G$ satisfies $\left({ }^{*}\right)$.

Proof. (i): Let $A<B \leqq G$ with $A$ maximal in $B$. If $A$ is not normal in $B$, let $a$ and $b$ be elements of $B$ with $[a, b]$ not in $A$. By $\left(^{*}\right)$ there is a subgroup $C \triangleleft B$ which does not contain both $a$ and $b$ but which contains $[a, b]$. Then $A \leqq A C \leqq B$ but $C \$ A$. Hence by the maximality of $A, A C=B$.

(ii): By Corollary 2, $G$ is an $S I^{*}$-group and from this fact it follows that $G$ satisfies $(Q)$ (see Kurosh [5, p. 183]). However, it is easy to give a proof which does not use the local theorem for SI-groups (which is needed for Corollary 2): Let $A<B \leqq G$ with $A$ maximal in $B$. Since the normal subgroups of $B$ satisfy the minimum condition, we can choose a minimal subgroup $K$ such that $K \triangleleft B$ and $B=A K$. Now $A \cap K \triangleleft A$; if $A \cap K \triangleleft K$, then $A \cap K \triangleleft B$. So assume that $A \cap K$ is not normal in $K$ and let $a$ and $b$ be elements $K$ such that $[a, b] \notin A \cap K$. By $\left({ }^{*}\right)$ there exists a subgroup $C$ of $K$ such that $C \triangleleft B,[a, b] \in C$, but not both $a$ and $b$ are in $C$. Hence $A<A C \leqq B$ since $[a, b] \notin A$. Thus $B=A C$ and the minimality of $K$ is contradicted.

(iii): Assume that the group $G$ satisfies the hypotheses of (iii) but does not satisfy $\left(^{*}\right)$. Let $H$ be a minimal subgroup of $G$ which does not satisfy $\left(^{*}\right)$. If $H$ is not finitely generated, all the finitely generated subgroups of $H$ satisfy $\left({ }^{*}\right)$; but this implies that $H$ satisfies $\left({ }^{*}\right)$ by Theorem 1 . Hence $H$ is finitely generated.

If $H$ contains a maximal subgroup $M$ which is normal, then $H / M$ is cyclic of prime order. Hence $M$ is finitely generated and satisfies $\left(^{*}\right)$ by the minimality of $H$. Therefore, by Theorem 2, $M$ is (finite and) solvable. But this implies that $H$ is solvable so that by Proposition $1, H$ satisfies $(*)$ - a contradiction.

So assume that every maximal subgroup of $H$ is not normal and let $A$ be a maximal subgroup of $H$. Then by $\left(Q^{\prime}\right)$ there is a proper normal subgroup $N$ of $H$ such that $H=N A$. Let $M$ be a maximal normal subgroup of $H$ containing $N$. Then $H=M A . H / M$ is simple and non-abelian. Also $H|M=M A| M \cong A \mid M \cap A$ so that $A$ has a non-abelian tor. But $A$ satisfies $\left({ }^{*}\right)$ since it is a proper subgroup of $H$, and hence by Lemma 2 , any tor of $A$ is abelian. Thus we have a contradiction and the theorem is proved.

COROLLARY 3. Let $G$ be a group which satisfies the minimum condition for subgroups. Then the following are equivalent:

(1) $G$ is solvable.

(2) G satisfies $\left({ }^{*}\right)$.

(3) $G$ satisfies $(Q)$.

(4) G satisfies $\left(Q^{\prime}\right)$. 
Proof. By Proposition 1, (1) implies (2). (2) implies (3) by Theorem 3 (ii). Clearly (3) implies (4). So assume (4). Then by Theorem 3 (iii) $G$ satisfies $(*)$. Hence by Corollary $2, G$ is an $S I^{*}$-group. Therefore, by a theorem of Cernikov, $G$ is solvable (see Kurosh [5, p. 191]).

REMARK: Since submitting this paper it has been drawn to my attention that Baer has two papers to appear shortly $([1]$ and [2]) in which he considers among other things the properties $(Q)$ and $\left(Q^{\prime}\right)$. The main theorem of [1] gives a number of criteria for a group $G$ to be artinian and solvable. One of these is:

(a) Abelian subgroups of $G$ are artinian.

(V) (b) If $F$ is a finitely generated subgroup of $G$, then

$\left(b^{\prime}\right)$ the normal subgroups of $F$ satisfy the minimum condition

and $\quad\left(b^{\prime \prime}\right)$ if $S$ is a maximal subgroup of $F, S$ is almost normal in $F$.

This criterion implies that if $G$ is artinian, then $G$ is solvable if, and only if $G$ satisfies $(Q)$. But, of course, it is a much stronger result.

In the same spirit we could prove: $G$ is artinian and solvable if, and only if

(a) Abelian subgroups of $G$ are artinian.

(b) If $F$ is a finitely generated subgroup of $G$, then

(b') the normal subgroups of $F$ satisfy the minimum condition

and $\left(b^{\prime \prime \prime}\right) F$ satisfies $\left(^{*}\right)$.

This follows from our Theorem 2 and the theorem of Cernikov (see [4]) which states: Let $G$ be locally finite and locally solvable. Then if abelian subgroups of $G$ are artinian, $G$ is artinian and solvable.

In Baer's paper 'Normalizatorreiche Gruppen' there is another proof of the fact that an artinian group $G$ is solvable if, and only if it satisfies $\left(Q^{\prime}\right)$ (see [2] Hilfsatz 3.6).

\section{The property $(* *)$}

Definition 6. The group $G$ satisfies (**) if: given an element $a(\neq 1)$ in $G$, there is a normal subgroup $N=N(a)$ of $G$ such that $[a, x] \in N \forall x \in G$ but $a \notin N$.

REMARK. If $G$ satisfies (**) and $a(\neq 1)$ is an element of $G$, we can define $N_{a}=\{\cap N \mid N \triangleleft G, a \notin N$ and $[a, x] \in N \forall x \in G\}$ then $N_{a} \triangleleft G, a \notin N_{a}$ and $[a, x] \in N_{a} . N_{a}$ is the unique smallest normal subgroup of $G$ with these properties.

As in the case of $\left(^{*}\right)$ we have:

LEMma 3. (i) If $S$ is a subgroup of the group $G$, and if $G$ satisfies (**), then $S$ satisfies $\left.{ }^{* *}\right)$. 
(ii) If $K$ is a normal subgroup of the group $G$, and if $G$ satisfies (**), then given an element $a(\neq 1)$ in $K$ there exists a normal subgroup $N$ of $G$ such that $N<K, a \notin N$ but $[a, x] \in N \forall x \in G$.

It is clear that $\left({ }^{* *}\right)$ implies $\left({ }^{*}\right)$. For if $a, b(\neq 1,1)$ are elements of the group $G$, then if $a \neq 1$ we can find a normal subgroup $N$ of $G$ such that $a \notin N$ but $[a, x] \in N$ for all $x \in G$. Thus $[a, b] \in N$ but not both $a$ and $b$ are in $N$. If $a=1, b \neq 1$ and we interchange the rôles of $a$ and $b$.

Proposition 3. If $G$ is a $Z$-group, then $G$ satisfies (**). In particular, if $G$ is nilpotent, $G$ satisfies (**).

Proof. Let $\Sigma$ be a central system for $G$. Let $a(\neq 1)$ be an element of $G$ and define

$$
\begin{aligned}
& \bar{N}=\{\cap K \mid K \in \Sigma, a \in K\} \\
& N=\{\cup L \mid L \in \Sigma, a \notin L\}
\end{aligned}
$$

Then $N<\bar{N}$ is a jump in $\Sigma$; hence $\bar{N} / N \leqq Z(G / N)$ and this implies that $[a, x] \in N \forall x \in G$.

Proposition 4. Let $G$ be a group and assume that for each element $a(\neq 1)$ a normal subgroup $N_{a}$ can be chosen so that $a \notin N_{a}$ but $[a, x] \in N_{a} \forall x \in G$ and that in addition these subgroups are linearly ordered. Then $G$ is a $Z$-group.

The proof of this proposition is quite similar to the proof of Proposition 2 and will be omitted.

THEOREM 4. If the group $G$ satisfies (**) locally, then $G$ satisfies (**).

Proof. Let $\Sigma$ consist of all finitely generated subgroups of $G$. For $H$ in $\Sigma$ and $a(\neq 1)$ in $H$ let $N_{a}(H)$ be a fixed normal subgroup of $H$ such that $a \notin N_{a}(H)$ but $[a, x) \in N_{a}(H) \forall x \in H$.

For $a(\neq 1)$ in $G$ and $S$ a finite subset of $G$ containing $a$, define $K_{a}(S)=\left\{\cap N_{a}(H) \mid H \in \Sigma, S \subseteq H\right\}$. Let $K_{a}=\left\{\cup K_{a}(S) \mid S\right.$ a finite subset of $G$ containing $a\}$. It is easy to verify that $K_{a}$ is a normal subgroup of $G$ such that $a \notin K_{a}$ but $[a, x] \in K_{a} \forall x \in G$.

LemmA 4. Let $G$ be a group which satisfies (**) and $Z$ a subgroup of the centre of $G$. Then $G / Z$ satisfies (**).

Proof. Let $a \in G \backslash Z$ and let $N_{a}$ be the minimal normal subgroup of $G$ such that $a \notin N_{a}$ but $[a, x] \in N_{a}, \forall x \in G$. Then $Z N_{a} \mid Z \triangleleft G / Z$ and $[Z a, Z x] \in Z N_{a} \mid Z, \forall x \in G$. We have to verify that $Z a \notin Z N_{a} \mid Z$.

So suppose that $a \in Z N_{a}$. Then we can write: $a=z c$, where $z \in Z$ and $c \in N_{a}$.

Now let $N_{c}$ be a normal subgroup of $G$ such that $c \notin N_{c}$, but $[c, x] \in N_{c} \forall x \in G$. Then $N_{\mathrm{c}} \cap N_{a} \triangleleft G$ and $N_{\mathrm{c}} \cap N_{a}<N_{a}$ since $c \notin N_{\mathrm{o}}$ but $c \in N_{a}$. Clearly $a \notin N_{c} \cap N_{a}$ since $a \notin N_{a}$. For any element $x \in G$, we have: 


$$
\begin{aligned}
{[a, x]=[z c, x] } & =[z, x]^{c}[c, x] \\
& =[c, x] \text { since } z \text { is a central element. }
\end{aligned}
$$

Hence $[a, x] \in N_{a} \cap N_{c}$, and the minimality of $N_{a}$ is contradicted. Thus $a \notin Z N_{a}$ so that $Z a \notin Z N_{a} / Z$.

Theorem 5. If the group $G$ satisfies (**) and if $C$ is the hypercentre of $G$, then $G / C$ satisfies (**).

Proof. We define the ascending central chain of $G$ by $Z_{0}=E$, $Z_{1}=Z(G), \cdots, Z_{\alpha+1} \mid Z_{\alpha}=Z\left(G / Z_{\alpha}\right)$ and $Z_{\alpha}=\left\{\cup Z_{\beta} \mid \beta<\alpha\right\}$ for $\alpha$ a limit ordinal. Then there is an ordinal $\nu$ such that $Z_{\nu}=Z_{\nu+1} . C=Z_{\nu}$ is the hypercentre of $G$.

We prove by transfinite induction that each $G / Z_{\alpha}$ satisfies (**). Clearly $G / Z_{0}$ satisfies $\left({ }^{* *}\right)$.

CASE 1. $\alpha=\beta+1$, and $G / Z_{\beta}$ satisfies (**). Then

$$
G / Z_{\alpha} \cong \frac{G / Z_{\beta}}{Z_{\beta+1} / Z_{\beta}}=\frac{G / Z_{\beta}}{Z\left(G / Z_{\beta}\right)}
$$

satisfies (**) by Lemma 4.

CASE 2. $\alpha$ is a limit ordinal, and $G / Z_{\beta}$ satisfies $\left({ }^{* *}\right)$ for $\beta<\alpha$. Thus if $a \in G \backslash Z_{\beta}$, there exists $U / Z_{\beta} \triangleleft G / Z_{\beta}$ such that $a \notin U$ but $[a, x] \in U$ for all $x$ in $G$. Hence $Z_{\beta} \leqq U \triangleleft G, a \notin U$ but $[a, x] \in U$ for all $x$ in $G$. Let

$$
V_{\beta}(a)=\left\{\cap U \mid Z_{\beta} \leqq U \triangleleft G, a \notin U,[a, x] \in U \forall x \in G\right\} .
$$

Then $Z_{\beta} \leqq V_{\beta}(a) \triangleright G, a \notin V_{\beta}(a)$ and $[a, x] \in V_{\beta}(a) \forall x \in G$, and $V_{\beta}(a)$ is the unique minimal subgroup of $G$ with these properties.

We verify that if $\beta \leqq \gamma<\alpha$ and $a \in G \backslash Z_{\gamma}$, then $V_{\beta}(a) \leqq V_{\gamma}(a)$. For $Z_{\beta} \leqq Z_{\gamma} \leqq V_{\gamma}(a), a \notin V_{\gamma}(a)$ and $[a, x] \in V_{\gamma}(a) \forall x \in G$. Hence by the minimality of $V_{\beta}(a), V_{\beta}(a) \leqq V_{\gamma}(a)$.

Now let $a \in G \backslash Z_{\alpha}$. Then $a \in G \backslash Z_{\beta}$ for all $\beta<\alpha$. Define $V(a)=$ $\left\{\cup V_{\beta}(a) \mid \beta<\alpha\right\}$. Since the $V_{\beta}(a)$ are linearly ordered and normal, $V(a)$ is a normal subgroup of $G ; a \notin V(a)$ but $[a, x] \in V(a) \forall x \in G$. Also, $Z_{\beta} \leqq V_{\beta}(a)$ for $\beta<\alpha \Rightarrow Z_{\alpha}=\cup Z_{\beta} \leqq \cup V_{\beta}(a)=V(a)$.

Hence $G / Z_{\alpha}$ satisfies (**) in this case also.

Lemma 5. If the group $G(\neq E)$ satisfies $\left({ }^{* *}\right)$ and the minimum condition for normal subgroups then for $E<H \triangleleft G, H \cap Z(G) \neq E$.

Proof. Let $N$ be a minimal normal subgroup of $G$ contained in $H$. If $N \$ Z(G)$, there are elements $a \in N$ and $x \in G$ such that $[a, x] \neq 1$. By (**) and Lemma 3 (ii) we can find a normal subgroup $N_{a}$ of $G$ such that $N_{a}<N, a \notin N_{a}$ but $[a, y] \in N_{a} \forall y \in G$. Hence $l \neq[a, x] \in N_{a}$ so that $E<N_{a}<N$ contrary to the minimality of $N$. Thus $N \leqq H \cap Z(G)$. 
THEOREM 6. If the group $G$ satisfies (**) and the minimum condition for normal subgroups, then $G$ is a $Z A$-group.

Proof. Let $C$ be the hypercentre of $G$. If $C \neq G, G / C$ satisfies (**) by Theorem 5. Hence since $G / C$ satisfies the minimum condition for normal subgroups, $Z(G / C) \neq E$ (provided $G \neq C$ ) by Lemma 5. But this is impossible. Hence $G=C$ and $G$ is a $Z A$-group.

COROLLARY 4. If $G$ is a finitely generated group satisfying (**) and the minimum condition for normal subgroups, then $G$ is finite and nilpotent.

Proof. By Theorem 2, $G$ is finite and by Theorem $6, G$ is a $Z A$-group. Hence $G$ is finite and nilpotent.

Finally we recall two further conditions which may be imposed on groups:

Definition 7. The group $G$ is an $N$-group if the normalizer condition holds in $G$, i.e. if every proper subgroup of $G$ is distinct from its normalizer.

Definition 8. A group $G$ is an $\tilde{N}$-group if in every subgroup $B$ of $G$ every maximal subgroup $A$ is normal.

THEOREM 7. Let $G$ be a group satisfying the minimum condition for subgroups. Then the following are equivalent:

(1) $G$ is a ZA-group.

(2) $G$ is an $N$-group.

(3) $G$ is an $\tilde{N}$-group.

(4) G satisfies (**).

(5) $G$ is locally finite and locally nilpotent.

Proof. It is well-known that (1) implies (2) (see e.g. Kurosh p. 215 and p. 219). A group $G$ is an $N$-group if and only if through each subgroup of $G$ there passes an ascending normal series, while $G$ is an $\tilde{N}$-group if for every subgroup of $G$ there is some normal system passing through it (see Kurosh pp. 220-21). Hence (3) follows from (2).

Now assume that $G$ is an $\tilde{N}$-group which does not satisfy $\left({ }^{* *}\right)$, and let $H$ be a minimal subgroup of $G$ which does not satisfy $\left({ }^{* *}\right)$. By Theorem 4, $H$ is finitely generated. Let $M$ be a maximal subgroup of $H$. Then $M$ is normal in $H$, and hence of finite index. Thus $M$ is a finitely generated subgroup of $G$ which satisfies (**). By Corollary 4, $M$ is finite. But this implies that $H$ is finite and a finite $\tilde{N}$-group is nilpotent (see Kurosh p. 216). Hence by Proposition 3, $H$ satisfies (**) - contrary to the choice of $H$. Therefore, (3) implies (4).

(5) follows from (4) by Corollary 4. Finally if $G$ satisfies (5), it is a $Z$-group and hence a $Z A$-group since it satisfies the minimum condition for subgroups. 
REMARK. It should be noted that the (equivalent) conditions in Theorem 7 do not imply that $G$ is nilpotent. For example, let $A$ be a group of type $p^{\infty}$ and let $B$ be cyclic of order $p$. Then $G=A$ wr $B$ (the wreath product of $A$ and $B$ ) is solvable and satisfies the minimum condition. Any finitely generated subgroup $H$ of $G$ is solvable and satisfies the minimum condition. Hence $H$ is a finite $p$-group and so nilpotent. Therefore, $G$ is locally nilpotent. But $G$ is not nilpotent since $A$ is not bounded (see Baumslag $[3, \S 3])$.

\section{References}

[1] Reinhold Baer, Soluble Artinian Groups (to appear).

[2] Reinhold Baer, Normalizatorreiche Gruppen (to appear).

[3] Gilbert Baumslag, 'Wreath Products and p-Groups', Proc. Cambridge Philos. Soc. 55 (1959), $224-31$.

[4] S. N. Cernikov, 'Über lokal auflösbare Gruppen, die dev Minimalbedingung genügen', Mat. Sbornik 28 (0000), $119-29$.

[5] A. G. Kurosh, The Theory of Groups II (English Translation, New York, 1956).

[6] D. H. McLain, 'On Locally Nilpotent Groups', Proc. Cambridge Philos. Soc. 52 (1956), $5-11$.

[7] O. Ore, 'Contributions to the theory of groups of finite order', Duke Math. J. 5 (1939), $431-460$.

Pennsylvania State University

University Park, Pennsylvania and

University of Frankfurt

Frankfurt am Main, Germany 